\title{
The Egalitarian Sharing Rule in Provision of Public Projects
}

\author{
Anna Bogomolnaia* $\quad$ Michel Le Breton ${ }^{\dagger} \quad$ Alexei Savvateev $^{\ddagger}$ \\ Shlomo Weber ${ }^{\S}$
}

\begin{abstract}
In this note we consider a society that partitions itself into disjoint jurisdictions, each choosing a location of its public project and a taxation scheme to finance it. The set of public project is multi-dimensional, and their costs could vary from jurisdiction to jurisdiction. We impose two principles, egalitarianism, that requires the equalization of the total cost for all agents in the same jurisdiction, and efficiency, that implies the minimization of the aggregate total cost within jurisdiction. We show that these two principles always yield a core-stable partition but a Nash stable partition may fail to exist.
\end{abstract}

Keywords: Jurisdictions, stable partitions, public projects, egalitarianism.

JEL Classification Numbers: C71, C72, D63, H41.

*Rice University, Houston, USA.

†Université de Toulouse I, GREMAQ and IDEI, Toulouse, France.

${ }^{\ddagger}$ Central Economics and Mathematics Institute, Moscow; Institute for Theoretical and Experimental Physics, Moscow; New Economic School, Moscow; CORE, Catholic University of Louvain-la-Neuve, Belgium. Financial support through grants R98-0631 from the Economic Education and Research Consortium, \# NSh-1939.2003.6 School Support, Russian Foundation for Basic Research No. 04-02-17227, and the Russian Science Support Foundation is gratefully acknowledged.

${ }^{\S}$ SMU, Dallas, USA, and CORE, Catholic University of Louvain, Belgium, and CEPR. 


\section{Introduction}

In this note we consider a model of jurisdiction formation, where the entire population has to be partitioned into several jurisdictions, each deciding on the public project from the multi-dimensional characteristics set. Whereas the existing literature deals with unidimensional universe of public projects, in this note we consider a set of projects to be imbedded in the set of an arbitrary dimension. Agents are assumed to have Euclidean preferences over possible project locations, and, thus, can be identified by their peaks (best preferred locations) in the multi-dimensional space.

When a jurisdiction is formed and a public project is selected, the jurisdiction must choose the way to distribute the project cost among its members. The discussion on the cost sharing methods in this context has progressed in two directions. One group of papers (Le Breton and Weber (2003), (2004), Haimanko et al. (2004a), Le Breton et al. (2004)) assumes the transferable utility framework with the unrestricted set of redistribution schemes within a jurisdiction. However, in many situations the degree of freedom in selecting a taxation scheme and degree of compensation to disadvantaged agents could be limited by customs, law, feasibility or variety of other constraints. Thus, a large number of papers (Alesina and Spolaore (1997), Casella (2001), Jéhiel and Scotchmer (2001), Haimanko et al. (2004b)) consider an equal share taxation scheme where all members of jurisdiction make an equal monetary contribution towards the projects costs. This cost sharing mechanism has some appealing properties, such as simplicity and anonymity. However, it rules out any degree of equalization between agents who enjoy vastly different benefits from the public project.

In this note we consider the case where every jurisdiction applies egalitarian rule of full equalization, when the total burden of taxation cost and disutility from having a project different from agent's ideal choice is equally divided among the members of the jurisdiction. The egalitarian rule in this context implements the Rawlsian principle by equalizing the total costs of all agents and, therefore, minimizing the total cost of the most disadvantaged agent. Under the egalitarian rule agents are not hold accountable for their preferences and the burden of total disutility is shifted to the entire jurisdiction.

The equal burden of all agents within every jurisdiction turns our model into hedonic game (Banerjee et al. (2001), Bogomolnaia and Jackson (2002)), where once a coalition is formed, one can uniquely determine the (equal) payoff of all of its members. Thus, agents form well-defined preferences over possible coalitions they could join. This feature of the hedonic model allows us to consider not only traditional cooperative stability notions (like core), but also non-cooperative stability, when only one agent can migrate to another

jurisdiction or create a new one. We show that whereas the core stable partitions always exist in our multi-dimensional framework, Nash stable partition may fail to exist even in the 
case where the set of projects is represented by a uni-dimensional space.

\section{The Model}

We consider a finite society $N=\{1, \ldots, n\}$ of agents. The set of public projects is given by the compact subset $I$ of $k$-dimensional Euclidean space $\Re_{k}$. Each agent has Euclidean preferences over $I$, which allows us to identify an agent $i$ with his/her ideal point $p^{i} \in I$. Slightly abusing the terminology, we will refer to $p^{i}$ as the location of the agent $i$.

The society faces a task of partitioning itself into disjoint jurisdictions, where each jurisdiction selects a location of the public project and a taxation scheme to finance it. We assume voluntary participation: the benefits from public projects exceed their costs. When the location of the project $p$ and a taxation scheme within jurisdiction $S$ are chosen, every member $i$ of $S$ incurs two types of cost: transportation cost from his/her location at $p^{i}$ to $p$ and the monetary contribution towards provision of the public project.

The locational and redistributional choices within each jurisdiction are guided by two principles, egalitarianism and efficiency. The egalitarian principle demands the equality of the total cost (transportation and project contribution) for all agents in the same jurisdiction. This requirement does, in fact, generate the Rawlsian allocation (see Le Breton et al. (2004)), that minimizes the total cost of the most disadvantaged agent within the jurisdiction. The efficiency requires the minimization of the aggregate total cost incurred by the members of the jurisdiction.

We assume that the cost of a public project in jurisdiction $S$ is given by the positive value $g(S)$, which may depend on the size and composition of a jurisdiction. The taxation scheme $x: S \rightarrow \Re$ assigns the contribution $x(i)$ for each agent $i \in S$. The only requirement is that the allocation $x$ must satisfy the budget constraint: $\sum_{i \in S} x(i)=g(S)$. In addition to the monetary contribution $x(i)$, each member of $S$ incurs disutility, or transportation cost, $d\left(\left\|p^{i}-p\right\|\right)$, where $p$ is the jurisdictional choice of the public project. We assume that function $d$ is continuous and increasing on $\Re$ with $d(0)=0$.

Given the location $p$ of the public project, the aggregate cost of members of jurisdiction $S$ will be the sum of the cost of the public project $g(S)$ and the aggregate transportation cost $D(S, p)$, where

$$
D(S, p)=\sum_{i \in S} d\left(\left\|p-p^{i}\right\|\right) .
$$

The efficiency requires that the project location is chosen in such a way as to minimize $D(S, p)$. Let

$$
D(S)=\min _{p \in I} D(S, p),
$$


and the location $p(S)$ (not necessarily uniquely defined) is determined by $D(S, p(S))=D(S)$.

Under the egalitarian taxation scheme, all members of $S$ equally share the aggregated total cost (tax plus transportation cost) $g(S)+D(S)$. Thus, every member $i$ of $S$ will incur the same cost $c(S)$ defined by

$$
c(S)=\frac{(g+D(S))}{|S|},
$$

where $|S|$ is the cardinality of jurisdiction $S$. Given that $i$ 's transportation cost is $d\left(\| p^{i}-\right.$ $p(S) \|$ ), her tax share (that can actually be a subsidy) should be

$$
\frac{(g(S)+D(S))}{|S|}-d\left(\left\|p^{i}-p(S)\right\|\right) .
$$

Efficiency and egalitarianism completely determine the choices for each potential jurisdiction. Thus, these requirements lead to hedonic cooperative coalition formation game, where once a jurisdiction is formed, the payoff or cost for each its member is uniquely determined. We will examine the existence of stable partitions in our hedonic game, where all jurisdictions adopt the egalitarian rule. We consider two notions of stability, where the first is the standard notion of the core of the coalition structure (Aumann and Drèze (1974)):

Definition 1: Let $\pi=\left\{S_{1}, \ldots, S_{K}\right\}$ be a jurisdictional structure. We say that a jurisdiction $S \subset N$ blocks $\pi$ if $c\left(S^{i}\right)>c(S)$ for all $i \in S$, where $S^{i}$ is the jurisdiction in $\pi$ that contains $i$.

A jurisdictional structure $\pi$ is called core-stable if no jurisdiction blocks $\pi$.

The second definition is that of Nash stability:

Definition 2: A jurisdictional structure $\pi=\left\{S_{k}\right\}_{k=1, \ldots, K}$ is Nash stable if

$$
c\left(S^{i}\right) \leq g(\{i\}) \text { and } c\left(S^{i}\right) \leq c\left(S_{k} \sqcup\{i\}\right)
$$

for for every $i \in S$ and every $S_{k} \in \pi$.

One can view a Nash stable jurisdictional structure as a free mobility equilibrium: no agent has an incentive to move from his/her current jurisdiction to either "empty" jurisdiction or to another existing jurisdiction. Note also that the set of Nash stable jurisdictional structures is the set of pure Nash equilibria of the non-cooperative game, where each agent announces his/her "address" and all agents with the same address form a jurisdiction.

While the definition of Nash stability allows only for deviations by a single agent, an agent can move to a jurisdiction without the consent of its members. Thus, some or even all members of that jurisdiction could be worse off. This observation shows that there is no logical connection between the notions of core- and Nash stability. And, indeed, the next section indicates sharply different stability implications with regard to these two notions. 


\section{The Results}

In the egalitarian game, all members of the same coalition bear the same total cost (or total disutility). Thus, each coalition $S$ is assigned a number $c(S)$ that represents the total cost of each of its members. Thus, all agents derive their preferences over coalitions from the common ordering by comparing jurisdictions on the basis of their assigned contributions. In hedonic games, such common ordering guarantees the existence of a stable partition. Our first proposition indeed states that, without any further qualification, stable jurisdictional structures always exist in the egalitarian game.

Proposition 1: The Egalitarian game always admits core-stable jurisdictional structures.

Proof $^{1}$ : Let us construct the partition $\pi$ as follows. Take a coalition $T^{1}$ that minimizes the contribution of its members across all coalitions:

$$
c\left(T^{1}\right)=\min _{S \subset N} c(S)
$$

(obviously, the choice of $T^{1}$ as well as of all subsequent elements of $\pi$ is not necessarily unique.)

If $T^{1}=N$, we are done. Otherwise, choose $T^{2} \subset N \backslash T^{1}$ to minimize the cost over all coalitions that have an empty intersection with $T^{1}$ :

$$
c\left(T^{2}\right)=\min _{S \subset N \backslash T^{1}} c(S)
$$

If $T^{1} \sqcup T^{2}=N$, we are done. Otherwise, choose $\left.T^{3} \subset N \backslash\left(T^{1} \sqcup T^{2}\right)\right)$ to minimize the cost over all coalitions that have an empty intersection with $T^{1} \sqcup T^{2}$ :

$$
c\left(T^{3}\right)=\min _{S \subset N \backslash\left(T^{1} \sqcup T^{2}\right)} c(S) .
$$

By continuing this process, after at most $n$ iterations, we obtain a partition $\pi=\left\{T^{1}, \ldots, T^{K}\right\}$ of $N$. We show that $\pi$ is stable. Indeed, consider an arbitrary coalition $T \subset N$, and let $T^{k}$ be the first coalition in $\pi$ that has a nonempty intersection with $T$, i.e., $k=\min \left\{j \mid T^{j} \bigcap T \neq \emptyset\right\}$. Then the choice of $T^{k}$ implies that $c\left(T^{k}\right) \leq c(T)$. Thus, no agent that belongs to both $T^{k}$ and $T$, is better off at $T$ as compared to $T^{k}$, which means that $T$ could not block $\pi$.

It is worthwhile pointing out that if all values $c(S)$ are different, our construction yields a unique outcome $\pi$. It is easy to demonstrate that $\pi$ is the unique core stable partition. Indeed, $T^{1}$ has to be a part of every stable partition, otherwise this very coalition will block

\footnotetext{
${ }^{1}$ Since the egalitarian game satisfies the common ranking property of Farrell and Scotchmer (1988), our result could be derived from theirs. However, we opted to offer a direct proof that also implies that if all values $c(S)$ are different, the core-stable partition is unique.
} 
the latter one; given that, $T^{2}$ must belong to it as well. By extending the argument, we obtain the uniqueness of the core stable partition in this case. $\square$

While we can guarantee core stability in the egalitarian game, such general statement does not hold for Nash stability:

Proposition 2: Under the Egalitarian rule a Nash stable jurisdictional structure may fail to exist even if the public project space is uni-dimensional.

Proof: Let space of public projects $I$ be given by the uni-dimensional interval $[0,4.1]$ and consider a society with six agents, located at points $p^{1}=0, p^{2}=p^{3}=1.9, p^{4}=p^{5}=p^{6}=4.1$. Let transportation costs be linear, i.e., $d\left(\left|p, p^{\prime}\right|\right)=\left|p-p^{\prime}\right|$ for all $p, p^{\prime} \in I$, and project costs $g(S)$ be equal to 1 for all jurisdictions $S$. We will show that under the Egalitarian rule, this society does not admit a Nash stable partition. Suppose, in negation, that $\pi$ is a Nash stable partition. Denote $T=\{4,5,6\}$. It is easy to verify that if there is $S \in \pi$ that contains one or two agents from $T$ in addition to some of agents $1,2,3$, then $c(S)>1$. Thus, if agents 4, 5, 6 are separated in at least two groups, they cannot be joined by other agents, implying that the members of $T$ should belong to the same jurisdiction in a Nash stable partition.

Since the members' cost exceeds 1 in every jurisdiction with more than four members, and agent 1 contributes strictly more than one in all multi-agent coalitions, except $\{1,2,3\}$, it follows that only candidates for Nash stable jurisdiction structures are:

$\{\{1,2,3\}, T\},\{\{1\},\{2\},\{3\} \sqcup T\},\{\{1\},\{3\},\{2\} \sqcup T\},\{\{1\},\{2,3\}, T\}$.

Consider the following cycle:

$\{\{1,2,3\}, T\} \rightarrow\{\{1,2\},\{3\} \sqcup T\} \rightarrow\{\{1\},\{2\},\{3\} \sqcup T\} \rightarrow\{\{1\},\{2,3\}, T\} \rightarrow\{\{1,2,3\}, T\}$, in which every partition is being obtained from the previous one by a shift of one of the dissatisfied agents to a jurisdiction that yields his/her a lower cost. Thus, no partition in the cycle is Nash stable, and due to a symmetry between agents 2 and 3, it rules out all candidates to constitute a Nash stable partition.

To conclude, let us notice that the only core-stable partition in this example is $\{\{1\},\{2,3\}, T\}$.

\section{References}

[1] Alesina, A. and E. Spolaore (1997), On the number and size of nations, Quarterly Journal of Economics 112, 1027-1056.

[2] Aumann, R.J. and J.H. Drèze (1974), Cooperative games with coalition structure, International Journal of Game Theory 3, 217-237. 
[3] Banerjee, S., Konishi, H., and T. Sönmez (2001), Core in a simple coalition formation game, Social Choice and Welfare 18, 135-153.

[4] Bogomolnaia, A. and M.O. Jackson (2002), The stability of hedonic coalition structures, Games and Economic Behavior 18, 201-230.

[5] Cassela, A. (2001), The role of market size in the formation of jurisdictions, Review of Economic Studies 68, 83-108.

[6] Farrell, J. and S. Scotchmer (1988), Partnerships, Quarterly Journal of Economics 279297.

[7] Greenberg, J. and S. Weber (1986), Strong Tiebout equilibrium under restricted preferences domain, Journal of Economic Theory 38, 101-117.

[8] Jéhiel, P. and S. Scotchmer (2001), Constitutional rules of exclusion in jurisdiction formation, Review of Economic Studies 68, 393-413.

[9] Haimanko, O., Le Breton, M. and S. Weber (2004a), Voluntary formation of communities for provision of public projects, Journal of Economic Theory 115, 1-34.

[10] Haimanko, O., Le Breton, M. and S. Weber (2004b), Transfers in a polarized country: bridging the gap between efficiency and stability, Journal of Public Economics, forthcoming.

[11] Le Breton, M. and S. Weber (2003), The art of making everybody happy: how to prevent a secession, IMF Stuff Papers, 50, 403-435.

[12] Le Breton, M. and S. Weber (2004), Secession-proof cost allocations and stable group structures in models of horizontal differentiation, in Group Formation in Economics: Networks, Clubs and Coalitions, Demange, G. and M. Wooders, eds., Cambridge University Press, 266-285.

[13] Le Breton, M., Weber, S. and J.H. Drèze (2004), The Rawlsian principle and secessionproofness in large heterogeneous societies, CORE Discussion Paper. 